\title{
The Pirouetted Premolar - A 180-degree Variation in a Tooth Position
}

\author{
Geon Pauly ${ }^{1 *}$, Abdul Malik ${ }^{2}$, Roopashri Rajesh Kashyap ${ }^{1}$, Raghavendra Kini ${ }^{1}$, Prasanna Kumar Rao ${ }^{1}$ and Gowri P \\ Bhandarkar ${ }^{1}$
}

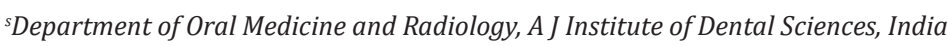

${ }^{2}$ Department of Orthodontics, H.K.E.S'S S. Nijalingappa Institute of Dental Sciences \& Research, India

*Corresponding author: Dr. Geon Pauly N, Postgraduate student, Department of Oral Medicine and Radiology, A J Institute of Dental Sciences, Kuntikana, NH-66. Mangaluru, PIN- 575004, Karnataka, India

Submission: 祭 February 27, 2018; Published: 眥 March 19, 2018

\begin{abstract}
Tooth rotation, is defined as mesio-lingual or disto-lingual intra-alveolar displacement of the tooth around its longitudinal axis. Such aberrations present a multi-factorial model in their origin. Both genetic and local factors seem to contribute to this abnormality. If the causative local factors and their contributions could be identified, a way could open up to an early treatment and prevention of secondary rotations of opposing and contiguous teeth. Herein, we discuss a case of a $180^{\circ}$ rotation of maxillary left second premolar in a female patient.
\end{abstract}

Keywords: Bicuspid; Rotation; Malocclusion

Case Report

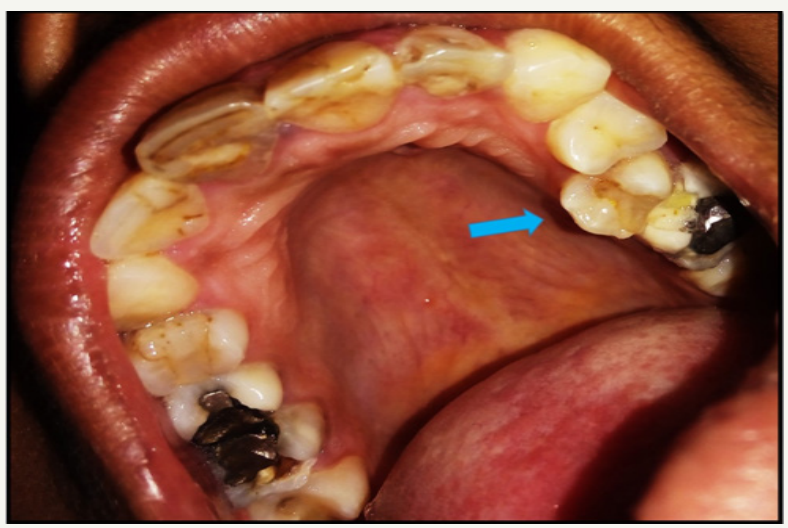

Figure 1: $180^{\circ}$ rotated maxillary second premolar on the left side.

A 35-year-old medically fit female patient reported to our department with a broken filling in upper left back tooth region. Her medical, family and personal histories were non-contributory and there was no sign of any systemic diseases or syndromes. On extra-oral examination, fractured amalgam restoration was seen in relation to left maxillary first molar which was non-tender to palpation and percussion. An additional incidental finding noticed was the adjacent second pre-molar which had been restored with composite restoration. The tooth was rotated a complete $180^{\circ}$ around its axis and placed slightly palatal to the line of the arch (Figure 1). The tooth was healthy and without any symptoms, and the rest of the teeth were in proper occlusion. The patient was made aware of such a positional abnormality in her mouth and referred for re-restoration in relation to the fractured restoration on the first molar.

\section{Discussion}

Tooth rotation, is defined as observable mesiolingual or distolingual intra-alveolar displacement of the tooth around its longitudinal axis [1]. Rotation of a tooth around an axis which is perpendicular to the occlusal plane through angles which approach $180^{\circ}$ is a rare anomaly which is found in the human dentition [2]. The aetiology of teeth rotations and displacements have been proposed by many authors over the years in various literatures. One possibility, of course, is that the premolars tooth crypts may have formed in the wrong positions and that the teeth's erupted malpositions may be reflecting this developmental anomaly [3]. Another theory that provides a reasonable validation is the 'theory of axial gradients'. It proposes that the polarity of a cell is determined by its metabolic rate. So likewise, the polo with the highest metabolic rate develops into the 'head', while the other one develops into the 'tails'. Putting this concept into effect for tooth having a $180^{\circ}$ rotation, it can be derived that via some 'accidents of nature', perhaps during the development of the tooth germ, the lingual portion grows at a faster rate than the buccal portion leading to the lingual portion of the tooth germ having grown into a 'buccal' cusp causing the positional variation [2].

Fixed appliances are unanimously considered the most effective treatment for such severely rotated teeth, like our case at hand. 
Also, in some conditions such as slight rotation, correction can be performed with a removable appliance such as an appliance with a labial bow and palatal spring [4]. One frequently encountered drawback is the relapse of the induced rotation, which is mainly because of the rebound of the elastic fibers in the supracrestal tissues. Though this side-effect can be reduced by pericision. The most common technique is the circumferential supracrestal fibrotomy, which involves inserting a surgical blade into the gingival sulcus to severe the epithelial attachment surrounding the involved teeth. It also results in cleaving of the transseptal fibers as the surgical blade enters interdentally into the periodontal ligament space. Usually, no surgical dressings are required and clinical healing usually is complete in 7-10 days [5].

\section{Conclusion}

It is often said, "What goes around comes around." Unfortunately, when it comes to rotated teeth, it ain't possible without dentists' interventions. The exact aetio-pathogenesis of tooth rotation is still not fully understood. And as we mentioned at the start, decoding the possible mechanism and causes could open up the door to an early treatment and thereby prevention of secondary rotations of opposing and contiguous teeth.

\section{References}

1. Parisay I, Boskabady M, Abdollahi M, Sufiani M (2014) Treatment of severe rotations of maxillary central incisors with whip appliance: Report of three cases. Dent Res J 11(1): 133-139.

2. Nayak G, Singh I (2013) A variation in tooth position- $180^{\circ}$ rotated maxillary second premolar. J Clin Diagn Res 7(8): 1806-1807.

3. Harris EF (2006) Commentary: rotated premolars. Dental Anthropology 19: 74-78.

4. Jahanbin A, Baghaii B, Parisay I (2010) Correction of a severely rotated maxillary central incisor with the whip device. Saudi Dent J 22(1): 41-44.

5. Shastri D, Tandon P, Singh GP, Singh A (2014) A new rotation correction technique: technique clinic. J Ind Orthod Soc 48(4): 566-569.
Creative Commons Attribution 4.0 International License

For possible submissions Click Here
Submit Article

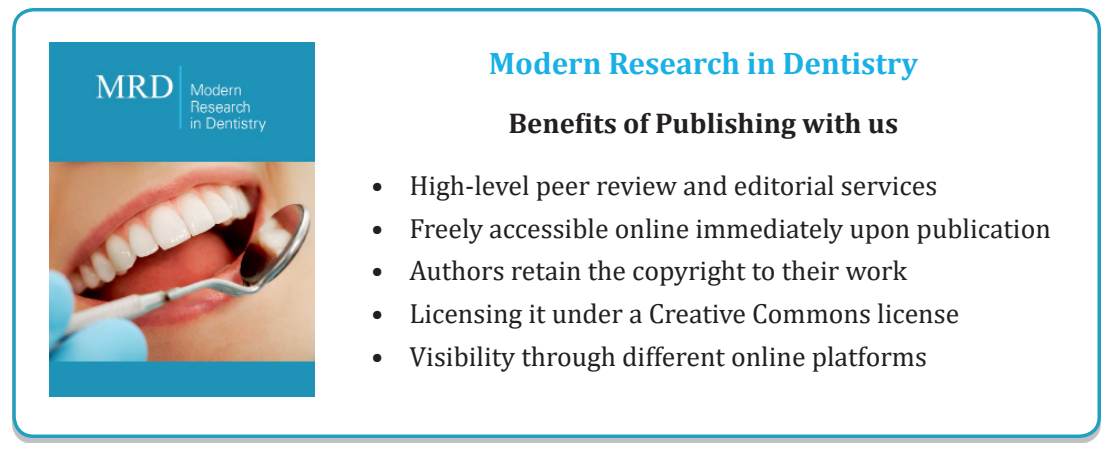

